\title{
Ribosomal proteins as novel players in tumorigenesis
}

de las Heras A., Perucho L., Paciucci R., Vilardell J, Lleonart ME.

1. Introduction

2. Ribosome biogenesis

3. Translational control

4. Oncogenes and tumor suppressor genes affecting ribosome biogenesis, translational control and protein synthesis

5. Extraribosomal functions of ribosomal proteins (RPs)

6. Ribosomal proteins as growth-promoting genes

7. Ribosomal proteins as growth-inhibitory genes

8. Ribosomal protein deficiency

9. Ribosomal protein transcripts

10. Clinical implications

11. Conclusions 


\begin{abstract}
Ribosome biogenesis is the most demanding energetic and metabolic expenditure of the cell. The nucleolus, a nuclear compartment, coordinates rRNA transcription, maturation and assembly into ribosome subunits. The transcription process is highly coordinated with ribosome biogenesis. In this context, ribosomal proteins (RPs) play a crucial role. In the last decade, an increasing number of studies have associated RPs with extraribosomal functions related to proliferation. Importantly, the expression of RPs appears to be deregulated in several human disorders due, at least in part, to genetic mutations. Although the deregulation of RPs in human malignancies is commonly observed, a more complex mechanism is believed to be involved, favoring the tumorigenic process. This review explores the roles of the most frequently mutated oncogenes and tumor suppressor genes in human cancer that modulate ribosome biogenesis, including their interaction with RPs. In this regard, we propose a new focus for novel therapies.
\end{abstract}




\section{Introduction}

The cell is the smallest metabolically functional unit of life; cells are able to sense and respond to external signals, a process that is tightly regulated at the transcriptional and translational levels. Translation is a highly coordinated multistep process in eukaryotic cells. The hardware of the translational machinery is composed of translation factors and ribosomes. Ribosomal components include ribosomal proteins (RPs) and rRNA. The biogenesis of eukaryotic ribosomes occurs within the nucleolus and requires the coordinated assembly of 4 different ribosomal RNAs (rRNAs) and 80 RPs $[1,2]$. Mammalian cells contain hundreds of copies of tandemly repeated ribosomal RNA (rRNA) genes that are transcribed with high efficiency to keep up with the cellular metabolic activity and demand for ribosomes. The human rRNA genes are organized into clusters of $43 \mathrm{~Kb}$ repeats that are distributed among different chromosomes. These gene clusters colocalize in the nucleolus, where rRNA synthesis and processing occurs. Ribosome biosynthesis monopolizes up to $80 \%$ of the transcriptional activity of rapidly growing cells through the transcription of the $5 \mathrm{~S}, 5.8 \mathrm{~S}, 18 \mathrm{~S}$ and $28 \mathrm{~S}$ ribosomal DNA (rDNA) genes. These rRNAs carry out essential structural and catalytic functions within the ribosomal core. The $18 \mathrm{~S}$ and $28 \mathrm{~S}$ genes are transcribed as immature polycistronic precursors by RNA polymerase I (Pol I) in the nucleolus, and the 5S and tRNA genes are synthesized by RNA polymerase III (Pol III) in the nucleoplasm. RPs are crucial for ribosome assembly and are synthesized by RNA polymerase II (Pol II). All three classes of RNA polymerases utilize auxiliary transcription factors to recognize specific gene promoters and initiate transcription. These complexes (holoenzymes) contain RNA polymerase, general transcription factors, coactivators, and proteins involved in protein modification, RNA chain elongation, RNA processing and DNA repair. This process is carefully coupled with the synthesis of RPs; after translation, the RPs migrate to the 
nucleolus for the assembly of $40 \mathrm{~S}$ and $60 \mathrm{~S}$ ribosomal subunits. The ribosome structure has revealed that many RPs may function as RNA chaperones during the assembly of ribosomal particles and/or in the stabilization of important domains of the rRNA [3]. Some RPs are involved in the interaction between the 40 and $60 \mathrm{~S}$ subunits (S13, S15, S19, L2, L5, L14), make contact with tRNA (S7, S9, S12, S13, L1, L5) or surround the polypeptide exit channel to stabilize the ribosome (L22, L24, L29). It had previously been assumed that most RP genes have a single gene expression control mechanism because they have common promoters and synonymous codon compositions. However, the modern ribosome can be defined as a ribonucleoprotein complex that can neither assemble nor function without its protein complement. Most RPs have been classified into subgroups according to their expression profiles, promoter structures and amino acid and codon compositions. Moreover, the specific expression of certain RP genes is associated with certain human tissues [4, 5]. It has been reported that RPs, in addition to their role in stabilizing the ribosome, also exhibit secondary functions that have not yet been fully characterized in other cellular processes such as DNA repair, apoptosis, drug resistance, proliferation, growth inhibition and chemoresistance $[6,7]$. For example, the RPs P0 and S3 possess apurinic/apyrimidinic endonuclease activity that strongly implicates these proteins in DNA repair functions or regulating the formation of specific RNA-binding complexes for selected mRNAs (Table 1) [8-10]. Most of the studies reporting that RPs have extraribosomal functions do not fully demonstrate that the indicated extraribosomal function is unrelated to the ribosome and protein translation. This review reinforces the results of a few studies that demonstrate that the extraribosomal function of RPs does not have an impact on the ribosome or protein synthesis in general terms. 
On the other hand, the traditional view is that cancer arises from DNA mutations or the deregulation of proteins involved in cell division. However, the importance of ribosome biogenesis and RPs in carcinogenesis has been overlooked. Several decades ago, it was observed that the inflated nucleoli of cancer cells represented a clear indication that rRNA synthesis is abnormally high [11]. Surprisingly, RPs have not been extensively characterized, despite their altered expression in some human tumors [12-15]. The great importance of ribosome function and translational control in tumor progression is underscored by the evidence that several oncogenes, such as Myc, and tumor suppressor genes, such as PTEN, pRb and p53, have been shown to directly regulate ribosome production and protein translation [16-18]. Moreover, the observation that several key oncogenes and tumor suppressor genes that are affected in cancer cells converge on a single point in modulating ribosome biogenesis and RPs suggests that the control of this process dictates cell fate. This review explores how ribosome biogenesis is ultimately coordinated with cell growth and proliferation. In particular, the role of RPs beyond ribosome biogenesis and protein synthesis is discussed to explore the potential of RPs as therapeutic targets in cancer.

\section{Ribosome biogenesis}

Ribosome biogenesis is a very important process in the life cycle of a cell and requires most of the activity of eukaryotic cells. rRNA transcription, rRNA processing, and ribosome assembly and transport require hundreds of accessory proteins (non-RPs) and approximately 200 small nucleolar RNA (snoRNAs) [19]. Examples of these non-RPs

are Transcription Initiation Factor I (TIF-IA), Selectivity factor 1 (SL-1), Upstream Binding Factor (UBF), Transcription Factor IIIB 90 kDa subunit (TFIIIB) and General Transcription Factor III C subunit alpha (TFIIIC). The snoRNAs are an abundant class of trans-acting RNAs that function in ribosome biogenesis in the eukaryotic nucleolus 
[20]. Most of the known snoRNAs guide the modification of pre-ribosomal RNA (prerRNA) by base pairing near target sites or are involved in cleavage of pre-rRNA by mechanisms that have not yet been described in detail. Interestingly, proliferation is inhibited when the snoRNA pathway is suppressed, and snoRNAs have been reported to be overexpressed in human breast and prostate cancer [21].

The biological mechanisms that control cell proliferation also control ribosome biogenesis. The rate of rRNA transcription is a limiting factor in the production of ribosomes, and controlling the synthesis of rRNAs is a way to regulate the translational capacity of cells. Moreover, rRNA synthesis is a limiting event for the proliferation of highly transformed cells. This situation is due in part to the fact that several potentially oncogenic transcription factors form protein complexes that bind to the promoter of the rRNA genes and initiate transcription. The importance of Pol I is demonstrated in yeast expressing a Pol I mutant that remains constitutively competent for the initiation of transcription under stress conditions [22]. The derepression of Pol I transcription leads to the derepression of Pol II transcription, which is restricted to the genes encoding RPs. Furthermore, the level of 5.8S rRNA, which is synthesized by Pol III, is deregulated concomitantly with Pol I transcription. A partial derepression of Pol I activity drives an abnormal accumulation of all ribosomal components, highlighting the critical role of the regulation of Pol I activity within the control of ribosome biogenesis [22]. Importantly, hyperactive ribosomal biogenesis, which has been attributed in part to increased rDNA transcription by Pol I, is widely observed in proliferative processes (dysplasia, hyperplasia) and cancer [23]. Among the auxiliary factors required by the DNAdependent RNA Pol I are TIF-IA, UBF and SL-1. TIF-IA is the mammalian homolog of yeast Rrn3p, a regulatory factor that is associated with the assembly of the preinitiation complex at the rDNA gene promoters (Figure 1) [24]. A dominant negative mutant of 
TIF-IA can suppress the progression of the cell cycle in proliferating HEK293T tumor cells, presumably by restricting ribosome production and halting growth [25]. In contrast, an activated version of TIF-IA that increases Pol I transcription is accompanied by proliferation activation. The inhibition of Pol I activity causes cell cycle arrest in $\mathrm{pRb}$ - and p53-proficient cells; removing this inhibition restarts the cell cycle progression [26]. In contrast, the inhibition of Pol I in pRb-and p53-deficient cells in which rRNA inhibition did not alter cell cycle progression causes a progressive ribosome depletion that leads to apoptotic cell death [26]. Moreover, the inhibition of rRNA transcription can induce apoptosis in cancer cells (but not normal cells) in tumors that are characterized by a high upregulation of ribosome biogenesis. For example, the antitumor activity of CX-5461, a potent small molecule inhibitor of rRNA synthesis, has been demonstrated in mice [27]. A disturbance in any single step in the process of ribosome biogenesis by distinct insults can lead to ribosomal stress (also called nucleolar stress). For example, BOP1, a protein localized to the nucleolus, has been shown to play a positive role in rRNA synthesis [28]. The inhibition of normal BOP1 function via the overexpression of a BOP1 mutant triggers a p53 response, suggesting that BOP1 may be a potential nucleolar integrator of stress signals. Ribosome biogenesis might be coordinated by multiple physiological processes (e.g., development and the circadian clock) and its dysregulation is closely associated with proliferation [29].

\section{Translation control}

The translation process is divided into four phases: initiation, elongation, termination and recycling. Initiation is the rate-limiting step and the best characterized step. Translation initiation is the process by which ribosomes are recruited to the 5' untranslated region $\left(5^{\prime}-\mathrm{UTR}\right)$ of mature mRNAs in the first step of protein synthesis. 
This process is highly regulated by a myriad of proteins that are designated as Eukaryotic Initiation Factors (eIFs). When translation begins, 40S ribosomal subunits are recruited to the $\mathrm{N}^{7}$-methyl guanosine cap ( $5^{\prime}$ cap) of mRNAs by the eIF4F translation initiation complex. eIF4F is composed of the cap-binding protein eIF4E, the helicase eIF4A and the scaffold eIF4G. The assembly of the eIF4F complex is limited by the abundance of eIF4E. Therefore, eIF4E is a limiting factor for translation initiation [30]. The majority of mRNA translation begins with eIF4F association with the cap; this process is known as cap-dependent translation and represents approximately $90 \%$ of the total translation in cells under standard conditions. Several mRNAs involved in the progression of the cell cycle have structured $5^{\prime}$-UTRs and depend on the eIF4F complex. Cells with overactivated eIF4F display neoplastic phenotype changes without significant changes in general translation. Instead, activated eIF4F dramatically stimulates the recruitment of ribosomes to a specific subset of mRNA-encoding proteins governing cancer-related functions [31]. At initiation, three steps occur: $43 \mathrm{~S}$ formation, $48 \mathrm{~S}$ formation and $80 \mathrm{~S}$ formation. $43 \mathrm{~S}$ formation occurs when the small $40 \mathrm{~S}$ subunit is loaded with the eIF2/Met-tRNA/GTP ternary complex, a process in which eIF2 activity is essential. $48 \mathrm{~S}$ formation occurs when the $43 \mathrm{~S}$ subunit is loaded with the mRNA with the assistance of the eIF4F complex. The ribosome then scans for the initiation codon and joins with the $60 \mathrm{~S}$ subunit to form the active $80 \mathrm{~S}$ ribosome.

The initiation of translation can also be regulated by additional mechanisms that involve internal ribosome entry sites (IRES) localized in the $5^{\prime}$ region of specific mRNAs. eIF4E controls the switch between cap-dependent and IRES-mediated translation [32]. IRES sequences permit the binding of an mRNA to $40 \mathrm{~S}$ ribosomes with limited assistance from the initiation factors. IRES-mediated translation represents 
approximately $10 \%$ of total translation under standard conditions but is activated under stress circumstances (such as proliferative disorders, apoptosis, viral infection, starvation, mitosis, and others) and becomes the main translation mechanism for certain mRNAs in the cell under these conditions. This arrangement allows cells to synthesize proteins when the cap-dependent pathway is inhibited [33]. For example, accurate mitotic progression is controlled by a general downregulation of cap-dependent translation, while the activation of IRES-dependent translation is needed to synthesize the mitotic proteins crucial for cytokinesis and centrosome replication. Of interest, Myc, p53 and $\mathrm{p} 27^{\mathrm{Kip} 1}$ contain IRES sequences at their $5^{\prime}$-UTR mRNAs, indicating the plasticity and adaptability of these proteins to certain physiological conditions $[34,35]$. Tumorigenic cells quickly adjust the rate of translation based on the availability of nutrients and growth-promoting mitogens and turn to IRES- dependent translation when cap-dependent translation is inhibited. In contrast, cells that exit the division cycle into a quiescent state greatly limit ribosome production and overall protein synthesis. The initiation of translation is an attractive potential target for pharmacological inhibition because it consists of specific steps regulated by eIFs under the control of oncogenic signaling.

Finally, a number of additional proteins that affect translation and mRNA stability, such us RNA-binding proteins, are mutated in specific cancer cells. This is the case for family with sequence similarity 46, member C (Fam46c) and DIS3 mitotic control homolog (Dis3) in multiple myeloma [36].

\section{Oncogenes and tumor suppressor genes affect ribosome biogenesis, translation control and protein synthesis}


Important oncogenes and tumor suppressor genes influence and modulate the key transcription factors involved in ribosome biogenesis. The most important of these genes will be discussed here.

\section{Oncogenes}

\section{a) $\mathrm{PI} 3 \mathrm{~K} / \mathrm{AKT}$}

The activation of the enzyme PI3K (phosphoinositide 3-kinase) via many growth factor receptors catalyzes the conversion of phosphatidylinositol $(4,5)$ bis-phosphate $\left[\mathrm{PI}(4,5) \mathrm{P}_{2}\right]$ to $\mathrm{PI}(3,4,5) \mathrm{P}_{3}$. PIP3 then recruits protein kinases that contain pleckstrin homology domains, including AKT, PDK1 and PDK2, to the plasma membrane. Increased PIP3 activates PDKs, which then in turn phosphorylate AKT at Thr-308 and Ser-473 [37]. The activation of AKT permits its release from the membrane and allows it to interact with and phosphorylate a range of cytoplasmic and nuclear substrates that ultimately promote cell survival. AKT is the main downstream target of PI3K that controls ribosome biogenesis, activates translation initiation and promotes translation elongation, ultimately enhancing protein synthesis, cell growth, cell cycle progression and survival (Figure 2). In particular, constitutive active AKT isoforms are sufficient to drive tumorigenesis, and the activation of the PI3K/AKT signaling pathway is crucial for the maintenance of cancer stem-like cells [38]. The PI3K/AKT pathway controls ribosome biogenesis by modulating the transcription of Pol I and Pol III [39]. Furthermore, in mammals, AKT controls translation initiation largely through the activation of its main substrate, mammalian target of rapamycin (mTOR), which promotes cell cycle progression and facilitates the translation of capped mRNAs. This process ultimately results in eIF4F activation. In fact, the primary regulation of eIF4F activity is exerted by the PI3K/AKT pathway. 
Moreover, AKT is also able to modulate IRES-mediated translation at the level of IRES-transacting factors (ITAFs). ITAFs bind to IRES sequences and play a pivotal role in IRES-containing mRNAs. It has been reported that the AKT pathway is essential for propagating the signal from Myc to the translational machinery by phosphorylating Mad1, a member of the Myc/Max/Mad family, and promoting its degradation [40]. AKT activity is elevated in many human tumors, and genetic lesions that affect the upstream components of AKT signaling are common in cancer [41]. For example, the loss of PTEN (the main inhibitor of PI3K/AKT) causes the upregulation of this pathway. PTEN is very frequently deleted or mutated in glioblastoma multiforme, endometrial cancer and advanced prostate cancer, in which the repressive action of PTEN on Pol I transcription may be lost by disrupting the SL-1 complex [42, 43].

b) $\mathrm{mTOR}$

The mTOR kinase regulates ribosome biogenesis at the levels of rRNA gene expression, 35S RNA processing, the transfer of the pre-ribosome from the nucleolus to the nucleoplasm, RPs and translation. Depending on its binding with different proteins, mTOR forms two main complexes, mTORC1 and mTORC2, with specific functions [44]. mTOR may initiate a signal leading to increased ribosome biogenesis, which is commonly observed in cancer cells. In both mammals and budding yeast, mTORC1 activates the transcription of Pol I by activating UBF and TIF-IA, which control ribosome biogenesis. In mouse cells, the mTOR pathway via S6K1 stimulates UBF phosphorylation in its C-terminal domain [45]. Moreover, the synthesis of tRNA and 5S rRNA by Pol III is regulated by the mTOR pathway, facilitating the association of the transcription factors TFIIIB and TFIIIC with 5S genes [46]. TFIIIB contains an mTOR signaling motif that interacts with mTOR, causing mTOR to localize to the tRNA and 
5S RNA genes, providing an opportunity for its direct control. By recruiting Pol III to its promoter templates, TFIIIB enhances the binding ability of another Pol III transcription factor, TFIIIC [47]. The repressor of Pol III transcription Mafl homolog (Mafl) is phosphorylated in an mTOR-dependent manner in cancer cells [48].

mTOR regulates the late stages of ribosome maturation via the nuclear GTP-binding protein Nog1 in S. cerevisiae. Nog1 forms part of a complex that includes 60S RPs and the pre-RPs Nop7 and Rlp24 [49]. This complex shuttles between the nucleolus and nucleoplasm and is tethered to the nucleolus by nutrient depletion and mTOR inactivation. The mTOR system and the Nog1 complex components, including Nop7 and Rlp24, are highly conserved from yeast to vertebrates. The Nop7 and Nog1 vertebrate homologues, known as Pescadillo Homolog 1, containing BRCT domain (PES1) and Nucleolar GTP-binding protein 1 (NGB/CRFG), respectively, are also involved in 60S ribosome maturation [49].

In addition, mTOR has direct control over translation via the eIFs family of proteins. mTORC1 phosphorylates S6 kinase 1/2 (S6K or p70(S6K)- $\alpha$ ) and the 4E-BP proteins. The 4E-BPs are a family of small proteins that compete with the transcription factor eIF4G for binding to the dorsal surface of the transcription factor eIF4E to regulate protein synthesis (Figure 2). Active hypophosphorylated 4E-BPs block eIF4E binding to the $5^{\prime}$-UTR of mRNAs in the eIF4F complex, thereby preventing eIF4G recruitment; phosphorylated 4E-BP1 correlates with survival in cancer [50]. Upon growth factor stimulation, 4E-BPs are phosphorylated by mTORC1, eIF4E is liberated, and capdependent translation is initiated. For example, the mTORC1-dependent activation of ribosomal proteins S6 kinases S6K1 and S6K2 leads to the phosphorylation of eIF4B and RPS6 [51, 52]. mTORC1 can also regulate translation elongation via the phosphorylation of eukaryotic elongation factor 2 (eEF2 $\alpha)$ and eEF2 kinase. 
Rapamycin, a specific mTOR inhibitor, causes the dephosphorylation of 4E-BPs, impairs the formation of eIF4F and inhibits the translation of specific mRNAs. The inhibition of mTOR signaling by rapamycin inactivates UBF and reduces its ability to associate with the basal rDNA transcription factor SL-1 [45].

Although mutations of mTOR have not been described in human cancers, its associated pathways, such as Ras, PTEN, AKT and PI3K, are commonly hyperactivated [53]. Novel mTOR competitive inhibitors are being developed because it is thought that rapamycin can lead to a feedback activation of AKT by mTORC2 that results in tumor outgrowth.

\section{c) The Ras/Raf/ERK pathway}

Ras kinase is activated upon stimulation by external factors during the cell cycle under normal conditions. In contrast, cancer cells carrying mutations in the Ras gene express a constitutively active Ras protein. The Ras-dependent mitogen-activated protein kinase (MAPKs) cascade plays a central role in the transduction of extracellular signals that affect gene expression. Among the MAPK family, the ERK1 and ERK2 kinases are the best characterized (Figure 3). Ras/Raf signaling has been shown to stimulate ribosome biogenesis and protein synthesis at three different levels: Pol I transcription, Pol III transcription and mRNA translation. Ras affects RNA Pol I and Pol III transcription through its main downstream molecules, MEKs and ERKs [39, 54]. For example, growth factors induce rRNA synthesis by activating the Ras-MAPK-dependent signaling cascade, which targets the Pol I-specific transcription initiation factor TIF-IA. Two serine residues of TIF-IA, Ser33 and Ser49, are phosphorylated by ERK and RSK kinases, activating this complex [25]. ERKs bind and phosphorylate TIF-IA and UBF and thus affect the transcription of the Pol I promoters and the Pol III-specific general 
transcription factor TFIIIB [55]. ERKs have several substrates, including the MNK family of kinases, which phosphorylate eIF4E. Moreover, the stimulation of Ras/ERK leads to the activation of the ribosomal S6 kinases RSK1 and RSK2, which have overlapping substrates with S6 kinases S6K1 and S6K29, including RPS6 and eIF4B. In addition, Ras acts through activated $\mathrm{C}$ kinase (PCL/PKC). eIF6 is phosphorylated by PKC $\beta$ II, which allows the joining of the $60 \mathrm{~S}$ subunit to form the $80 \mathrm{~S}$ complex and translation initiation [56]. The mutation of the PKC $\beta$ II phosphorylation site of eIF6 reduces tumor growth in mice [56]. Ultimately, the control of eIF6 is necessary for maintaining ribosome biogenesis and high levels of translation (i.e., upon mitogenic stimulation) [56].

By activating ERKs, Ras can stabilize Myc by phosphorylation (at Ser62) and block Myc degradation by inhibiting its phosphorylation (at Thr58) by GSK3 $\beta[40,57]$. Through ERK or PI3K signaling, Ras can lead to cellular transformation by altering the transcriptome, producing a radical shift in the composition of mRNAs associated with actively translating polysomes [58].

The Ras/Raf/ERK signaling cascade is highly altered in human tumors; mutations in Ras are the most common mutations in colon and lung carcinomas and malignant cell lines [59]. In addition, ERK is hyperactive (hyperphosphorylated) in $30 \%$ of human tumors. It has been recently reported that mutations in the Ras pathway induce rapamycin-resistance and tumor proliferation [60]. Importantly, cancer patients with Ras mutations respond favorably to EGFR inhibitors. The consequences of this response at the transcriptional level are still unknown and may have important therapeutic implications.

d) $\mathrm{Myc}$ 
Myc is an essential protein that coordinates a transcriptional program driving cell growth and division and regulates a large number of genes, including several components of the protein synthesis pathway. Genes regulated by Myc include glucose metabolism factors, mitochondrial homeostasis factors, ribosomal biogenesis factors, RP genes, amino acid metabolism genes, translation initiation factors, iron metabolism genes, differentiation, and apoptosis and proliferation factors [61]. Importantly, Myc is regulated by two Ras effector pathways: the extracellular signal-regulated kinase (ERK) and phosphatidylinositol 3-kinase (PI3K) pathways [57]. It has been shown that at least a low level of Myc expression is detectable in the nucleolus after serum induction of starved cells, indicating a possible interaction with the ribosome [62]. rRNA synthesis and accumulation occur rapidly following the activation of a conditional Myc-ER allele [62]. In addition, Myc activates the transcription of Pol I, Pol II and Pol III [63]. For example, Myc binds to specific consensus elements located in human rDNA and associates with the Pol I-specific factor SL-1, directly enhancing Pol I transcription [62]. Endogenous Myc has been reported to be associated with tRNA and 5S rRNA genes. Moreover, Transformation/Transcriptional Domain-Associated Protein (TRRAP) and Histone Acetyltransferase PCAF (GCN5) are used by Myc to activate Pol III transcription [64]. Part of the ability of Myc to stimulate rRNA synthesis is due to the induction of UBF expression [65]. In an attempt to identify the Myc target genes that are involved in rRNA processing, Schlosser et al., showed that Myc upregulates the expression of PES1, Block of proliferation 1 protein (BOP1) and mammalian WD repeat protein 12 (WDR12) [66]. These three proteins interact with each other, forming a major complex (known as the PeBow complex) that is involved in the control of ribosome biogenesis and cell cycle ( $\mathrm{S}$ phase entry); the integrity of this complex is required for ribosome biogenesis and cell proliferation [67]. 
A role for Myc as a general inducer of protein synthesis is suggested by the finding that it induces the translation of eIF4E, eIF-2- $\alpha$, eIF5A and eIF4G [68]. Moreover, Myc and mTOR converge on a common node in protein synthesis control; the mTOR-dependent phosphorylation of 4EB-P1 is required for cancer cell survival in Myc-dependent tumor initiation and maintenance [69]. Importantly, an inhibitor that is capable of blocking mTOR-dependent 4EB-P1 phosphorylation has remarkable therapeutic efficacy in Mycdriven hematological cancers [69].

On the other hand, Myc is closely linked to several RPs, and certain RPs are able to regulate its activity. It has been reported that the RPs S14, L5 and L11 interact with Myc to inactivate it, causing cell growth arrest or apoptosis as a consequence. For example, L11 recruits miR-24/mRISC to repress Myc expression in response to ribosomal stress [70]. Moreover, L11 associates with Myc at 5S rRNA and tRNA genes and regulates their expression [71]. Another RP, S14, interacts with the homology box II (MBII) and the C-terminal basic helix-loop-helix leucine zipper (bHLH-LZ) domains of Myc [72]. S14 inhibits Myc transcriptional activity by preventing its recruitment (and the recruitment of its cofactor, TRRAP) to target gene promoters, thus suppressing Myc-induced cell proliferation [73]. Interestingly, the ectopic expression of Myc results in the increased expression of the genes encoding several RPs, such as S3, L3, L6, L23, L35 and L44 [74]. Importantly, Myc oncogenic activity can be suppressed by RP haploinsufficiency [75]. Moreover, L11 inhibits Myc but stimulates p53 transcriptional activity [76]. In addition, the deregulation of translational control due to Myc hyperactivation leads to genomic instability by modulating the translation of specific mRNAs. For example, Myc oncogenic signaling hyperactivates the expression of L24, prevents the switch to IRES-mediated translation in mitosis, and induces cytokinetic defects, genome instability and cancer initiation [75]. The RP L23 is a direct target gene 
that is transactivated by Myc and a negative regulator of the c-myc-associated zincfinger protein Miz-1 [77].

The deregulation of Myc is a common feature of some tumor types such as Burkitt's lymphomas, neuroblastomas and colon carcinomas [78]. Researchers have devoted much effort to studying the proteins and transcription factors that are involved in cell division. However, the mechanisms that link the key checkpoints in metabolism with ribosomal biogenesis and RPs with proliferation are very poorly understood. In addition to its broad role as a transcription factor, Myc is the only protein that directly modulates ribosome biogenesis, protein synthesis and proliferation at all levels. This fact suggests that Myc is a central mediator that coordinates all processes.

\section{Tumor suppressor genes}

e) $\mathrm{Rb}$

The $\mathrm{Rb}$ family of proteins $\mathrm{pRb} 1 / 105, \mathrm{p} 107$, and $\mathrm{pRb} 2 / \mathrm{p} 130$ are collectively referred to as pocket proteins. In addition to the well-defined role of inhibiting the G1 phase of the cell cycle, active Rb (non-phosphorylated) inhibits rRNA synthesis by binding to UBF and inhibits Pol III transcription by binding to TFIIIB $[79,80]$. Remarkably, in dividing cells, $\mathrm{Rb}$ phosphorylation facilitates cell cycle progression by releasing $\mathrm{E} 2 \mathrm{~F}$ and induces increased rRNA transcription. In this context, Rb regulates Pol I and Pol III, and as a result, cells derived from $\mathrm{Rb}$ knock-out mice have diminished Pol III activity [80]. However, Pol I activity increases only if p130 is ablated. The interactions between $\mathrm{pRb}$ and p130 have been shown to impair UBF functions, such as DNA binding or the interaction with SL-1. Known oncogenes, such as the E7 protein of human papillomavirus, adenoviral E1a protein and polyomavirus large T antigen, neutralize the pocket proteins and derepress Pol III transcription; Pol III in turn regulates 5S rRNA, 
tRNA and snoRNAs, which are required for ribosome biogenesis. The loss of Rb function is a common alteration in several cancers, including retinoblastoma, where it was first discovered [81]. $\mathrm{Rb}$ mutations strongly reduce the ability of $\mathrm{pRb}$ to negatively control rRNA transcription. pRb2/p130 is rarely compromised in cancer but is targeted by cyclin-dependent kinases, leading to their hyperphosphorylation and preventing its interaction with UBF and TIFIIIB.

f) p53

The tumor suppressor p53 has been proposed as the guardian of the genome [82]. The inactivation of p53 results in a failure to respond properly to several stress signals, leading to genomic instability and eventually cancer. The best known and characterized $\mathrm{p} 53$ downstream effect is the activation of $\mathrm{p} 21^{\mathrm{WAF} 1 / \mathrm{CIP} 1}$, a cell cycle inhibitor whose induction provokes cell cycle arrest [82]. Moreover, p53 activates murine double minute 2 protein (MDM2 or HDM2 in humans) to control its own degradation. In turn, the main p53 regulator is MDM2. MDM2 controls p53 activity by binding to p53 and interfering with its transactivation activity and by facilitating the proteasomal degradation of p53 by acting as an E3 ubiquitin ligase. Interestingly, the nucleolus is a common stress sensor of pivotal importance in the p53 response. In addition to regulating genes that control cell cycle arrest or apoptosis, p53 controls ribosomal biogenesis [83]. p53 activates or represses the transcription of several viral and cellular promoters transcribed by Pol I, Pol II and Pol III, including genes encoding a variety of non-translated RNAs that play critical roles at numerous stages during global gene expression $[84,85]$. Interestingly, the ectopic expression of wild-type p53 in HeLa cells results in a dramatic decrease in Pol I transcriptional activity by interfering with the formation of an initiation complex that includes SL-1 [85]. Moreover, p53 inhibits Pol 
III transcription by binding to the transcription factor TFIIIB. p53 is also directly involved in the transcriptional regulation of human U1 and U6 snoRNAs by both Pol II and III [86]. U1 and U6 snoRNA transcription is downregulated in MCF-7 cells after UV exposure and p53 activation. The fact that the U1 and U6 snoRNA genes contain a high-affinity p53 binding element located within the core promoter region suggests that p53 uses a different mechanism to repress U1 and U6 transcription [87]. In addition, the regulation of the 4EB-P1/eIF4E system constitutes a physiologically relevant aspect of proliferation inhibition by $\mathrm{p} 53$, which is independent of its role in apoptosis [88].

Lastly, the relationship between p53 and RPs will be discussed elsewhere (see section 8). More than $50 \%$ of human cancers harbor p53 mutations. P53 mutations confer a gain of function/oncogenic function that is separate from the sequestration of wild-type p53. Interestingly, by searching for possible p53 novel targets, it was found that RPs S2, P1 and L37 were overexpressed in the presence of mutated P53 (Arg to Trp in codon 248) [89]. Given the fact that p53 has more than one hundred target genes, it is likely that at least some RP genes have remained unnoticed as possible p53 target proteins until now. A detailed study of such interactions would be of extreme interest.

\section{g) INK4A-ARF}

The INK4A-ARF locus encodes two tumor suppressor proteins, $\mathrm{p} 16^{\mathrm{INK} 4 \mathrm{~A}}$ and $\mathrm{p} 19^{\mathrm{ARF}}$ ( $\mathrm{p} 14^{\mathrm{ARF}}$ in humans), that activate the growth suppressor functions of $\mathrm{Rb}$ and $\mathrm{p} 53$, respectively. ARF controls ribosome biogenesis by regulating the Pol I transcription factor designated Transcription termination factor 1 (TTF-1) [90]. The role of ARF in ribosome biogenesis is closely associated with Nucleophosmin (NPM, B23, nutramin or NO38) [91]. NPM is an abundant nuclear phosphoprotein that is involved in multiple cellular functions, including pre-rRNA processing, ribosome assembly, ribosome 
transport, centrosome duplication, molecular chaperone activity and regulation of HDM2-p53 activity. Specifically, NPM binds to HDM2 and acts as a negative regulator of the p53-HDM2 interaction, establishing a link between ribosome biogenesis and p53 [92]. It has been demonstrated that ARF physically interacts with NPM and inhibits NPM by proteasomal degradation [93]. The functional integrity of the core motif of NPM is required for stability, efficient nucleolar localization as well as ARF binding [91]. In addition to activating p53 by interacting with the MDM2-p53 pathway (ARFMDM2-p53) [93], p19 ${ }^{\mathrm{ARF}}$ can interact with NPM and partially inhibit the cleavage of 32S RNA; whereas p14 ${ }^{\mathrm{ARF}}$ has been shown to participate in the degradation of NPM by the proteasome. In fact, proliferation can be stimulated in tumor cells by overexpressing NPM. Moreover, UBF is a partner of $\mathrm{p} 14^{\mathrm{ARF}}$ in the transcription machinery by $\mathrm{p} 53$ independent mechanisms [94], and DDX5 helicase, a p53-independent target of ARF, participates in ribosome biogenesis [95]. These studies establish a previously unidentified nucleolar function for ARF in inhibiting ribosomal biogenesis and modulating growth arrest upon oncogenic insult; this effect is independent of the ARFMDM2-p53 pathway.

Importantly, the tumor suppressor $\mathrm{p} 14^{\mathrm{ARF}}$ is mutated in $40 \%$ of human tumors, and its loss may be responsible for the enhancement of ribosome biogenesis directly and indirectly through its effect on p53 stabilization [96].

\section{Extraribosomal functions of RPs}

It is worth mentioning that RP nomenclature has been confusing for some time. It seemed that each model system (or laboratory) had its own rules for naming RPs. For example, L30 has been known in the past in yeast as L30e, L32, rp73, or YL38 (www.yeastgenome.org). While efforts have been made to standardize RP names based 
on the mammalian ribosome, this confusion has to be taken into account, especially when reviewing literature from the last century [97, 98].

To make clear statements, it is necessary to define what exactly an extraribosomal function of an RP is. A previous report established the following criteria: 1) a demonstration that the RP in question interacts specifically with some non-ribosomal component of the cells, presumably RNA or protein; 2) a demonstration that such an interaction has a physiological effect on a living (or dying) cell; and 3) evidence that the physiological effect occurs away from the ribosome [99]. We suggest that these criteria should be refined by including one additional criterion: 4) a demonstration that the nonribosomal function does not influence the ribosome and does not directly affect translation and protein synthesis in the cell. We would like to remark that the second and fourth criteria have to be considered with caution because the demonstration of both criteria can be very difficult, as the depletion or knock-out of an RP can affect its action with the ribosomes and provoke a "background noise" that makes the confirmation of an extraribosomal function difficult. Therefore, the second and fourth criteria must be somewhat loosely considered.

Instead, there are a number of indirect observations that serve as good evidence that RPs can, in general, have extraribosomal functions: i) Mammalian cells make a substantial excess of RPs, which are subsequently degraded [100], and ii) most RP genes in yeast have two genes that encode identical or very similar amino acid sequences. In most cases, both genes are active; either alone can support growth at nearly normal to somewhat slower rates. Importantly, genome-wide screens have identified deletions of specific RP genes as influencing a number of situations, such as life span [101]. iii) Unlike mammals, plants have several genes for most RPs, many of which are developmentally regulated [102]. Overall, while these indirect observations suggest 
with high probability that certain RPs have extraribosomal functions, the direct demonstrations are less numerous and more difficult to obtain depending on the type of extraribosomal function under study. Some examples of evident extraribosomal functions are mentioned below. For example, yeast cells that are deficient in RP L1 (L10A in mammals) produce ribosomes lacking L1 that are exported to the cytoplasm and can be incorporated into polyribosomes. The presence of such defective ribosomes leads to slow growth and renders the cells hypersensitive to lesions in the ubiquitinproteasome system [103]. The observation that yeast cells cannot grow efficiently without L1 does not necessarily imply that translation cannot occur on ribosomes lacking L1; it simply occurs at a reduced rate. In human cells, L13A constitutes a good example. L13A is a RP that is susceptible to be phosphorylation; upon phosphorylation, L13A releases from the ribosome and join to the GAIT complex, where several enzymes converge to inhibit the translation of specific mRNAs by binding to a structure in their 3 '-UTR [104]. The ribosomes lacking L13A continue to translate mRNA and the cell continues to grow at a normal rate [104]. In mammalian HEK293 cells, the downregulation of $\mathrm{P} 1$ and $\mathrm{P} 2$ causes an altered polysome pattern with reduced translation efficiency, but without affecting the free $40 \mathrm{~S} / 60 \mathrm{~S}$ subunit ratio [105]. Upon $\mathrm{P} 1$ and $\mathrm{P} 2$ downregulation, the most prominent effect is a reduction in the proliferative capacity of the cells.

In vivo, mice embryos that are mutant for the RP L38 show alterations in the translational control of a subset of Hox genes [106]. Importantly, these mice do not have changes in global protein synthesis and do not have noticeable differences in the ratio of small to large ribosome subunits or polysome distribution compared to wildtype mice [106]. In the same line of evidence, preliminary experiments from our 
laboratory suggest that the absence of an RP does not have a significant effect on protein synthesis.

On the other hand, of particular interest is the RP named Receptor for activated $\mathrm{C}$ kinase (RACK1). RACK1 was originally identified based on its ability to bind to the PKC isoform $\beta$ II [107]. As a member of the Trp-Asp (WD) repeat protein family, RACK1 is a scaffold protein for many kinases and receptors and plays a pivotal role in signal transduction, cell growth, migration and differentiation. Recent studies have shown that RACK1 is also a component of the ribosomal 40S subunit, and its presence in both ribosome and non-ribosome bound forms is still matter of debate $[108,109]$. In response to stress conditions, RACK1 can be found in stress granules, multimolecular structures in the cytoplasm of stressed cells that contain stalled translation initiation complexes [110]. In these structures, RACK1 inhibits the apoptosis induced by the SAPK pathway. RACK1 was shown to be part of a messenger ribonucleoprotein complex bound to poly-A mRNAs in neurons. Recently, RACK1 has been defined as a "ribosome receptor," representing a docking site on ribosomes for the $\beta$-actin complex with the RNA-binding protein ZBP1 [111]. The binding of RACK1 to this complex is critical for the release and translation of $\beta$-actin mRNA [111]. In addition, RACK1 levels are critical for general protein translation, as observed by the very significant decrease in polysome formation in cells with knocked down RACK1 [112]. RACK1 protein levels are critical for the PTOV1-promoted translation of c-Jun mRNA in prostate cancer cells [112]. Although the role of RACK1 in protein synthesis is not completely clear, the above findings suggest that it is required for the translation of mRNAs, possibly providing a scaffold to accommodate translation regulation by different signaling pathways $[112,113]$. It is worthwhile to mention that in response to IGF1 or PMA, RACK1 rapidly colocalizes with RPS6 at submembrane sites and, 
similarly to the RP S6, RACK1 is not found in soluble fractions by sucrose gradient analysis [112]. These observations suggest that the role of RACK1 in signal transduction is linked to ribosome association. Moreover, RACK1 coupling to PKC $\beta$ II promotes the phosphorylation of eIF4E, stimulating survival [114].

In human tumors, RACK1 is upregulated and considered an excellent marker of oral squamous carcinoma, breast and lung cancer [115]. Recently, RACK1 was found to be upregulated in hepatocellular carcinoma, and its aberrant expression was shown to contribute to chemoresistance and tumor growth. Remarkably, these effects depended on the ribosome localization of RACK1 [114]. Therefore, RACK1 and other proteins that are clearly associated with ribosome activity but have no completely defined function have a key role in growth and survival and may have great future implications in the clinic.

\section{RPs as growth-promoting proteins}

The extraribosomal function of some RPs related to proliferation (even in normal cells) is being established as a new concept in biology $[116,117]$. In numerous cancers, it has been found that protein synthesis rates and the expression of several translation components are often significantly elevated [118]. It is generally assumed that this elevation is due to the higher proliferative rate of cancer cells. Under this scenario, the most logical belief is that the upregulation of RPs that is detected in human tumors is an indirect consequence of proliferation. Experimental evidence suggests that there is upand down-regulation of several RPs in the same tumor and that these changes do not necessarily involve the up or down-regulation of all RPs in an orchestrated mechanism. Moreover, increasing evidence suggests a direct association between RPs and cell growth and transformation. The discovery that varying the expression of an RP gene 
altered cellular responses affecting cell growth raised the question: Are the changes in RP expression a result of tumorigenesis or a cause of it? While the simple answer for decades was the former, new findings are causing many to revisit this old question. The study of the overexpression of a ribosomal protein in a wild-type context has been extremely useful. Many genetic screens have discovered immortalizing genes by examining their capacity to bypass replicative senescence in primary murine cells [119]. The mouse embryonic fibroblast (MEF) model has been particularly useful in these studies because a single genetic alteration in a growth-promoting gene is able to immortalize these cells. Previous results from our laboratory have demonstrated that the overexpression of $\mathrm{P} 1$, which belongs to the $\mathrm{P}$ protein group, bypasses senescence, immortalizes primary cells and is involved in transformation [120]. Importantly, the mRNA levels of P0, P1 and P2 (P Proteins) are increased in different cancer types, suggesting that these proteins might have a relevant role in tumorigenesis. In particular, P proteins represent prognosis markers in specific ovarian tumors [121]. The oncogenic role of $\mathrm{P} 0$ in promoting cell growth and colony formation has also been demonstrated in breast and hepatocellular cancer cell lines [122]. The RP L10, also known as QM, is an apparent Jun-binding/inhibitory protein that can be translocated to the nucleus after interaction with presenilin-1 protein [123]. The overexpression of other RPs, such as S3A, induces the transformation of NIH3T3 cells, as measured by well-established criteria such as focus formation, anchorage-independent growth in vitro and the formation of tumors in nude mice [124]. The physiological role of S3 is puzzling because genomic damage can lead to a dose-dependent transfer of S3 from the cytoplasm to the nucleus as a result of ERK-mediated phosphorylation [125]. RPS3 can also enter the nucleus and become part of the NFKB complex in response to TNF [126]. The silencing of the RPs L26 and L29 by RNA interference inhibits the proliferation of 
human PANC-1 pancreatic cells, suggesting a function of these RPs related to proliferation [127]. In the same line of evidence, siRNA experiments show that the silencing of L19 abrogates the aggressive phenotype of human prostate cancer [128]. The interaction between RPs and oncogenes and tumor suppressor genes is increasingly being reported. For example, S13 promotes gastric cancer growth through the downregulation of $\mathrm{p} 27^{\mathrm{Kip} 1}$ [129]. Many RPs have been found to be upregulated in human cancers and cell lines compared to their normal counterparts or parental cell lines (Table 2) [130-132]. Several RPs are overexpressed upon oncogenic stimulation, as described earlier for the case of Myc and p53. Moreover, some transcription factors, such as NF- $\alpha 1$ (EF2), NF- $\beta 1$, GABP or nuclear respiratory factor 2 , have been reported to interact with and regulate the expression of specific RPs by binding to their promoters, as is the case of the RP genes rpL30 and rpL32 [133]. In addition to the various RP genes that are upregulated in human tumors [12], a limited number of RPs have been proposed as prognostic or diagnostic markers in various cancer types. For example, the expression of L21 and L16 has been associated with metastatic behavior when comparing the genetic proliferation of metastatic versus non-metastatic nasopharyngeal carcinoma cell lines [134]. In addition, L19 has been found to be a predictor of prostate cancer progression [14], L13 upregulation correlates with clinical staging in gastric cancers [135] and S27-like expression improves the prognoses of colon cancer patients [136]. In a limited series of patients with benign tumors (adenomas), the overexpression of S3, S8, S12, L5, S6, and P0 has been described [137]. Although this series is insufficient to establish any conclusion, it is striking that RPs were upregulated in 7 out of 10 pre-malignant adenomas compared to normal mucosa. Remarkably, this increase also occurs in the cancerous tissue [137]. In the particular case of the colonic tissue, it is unlikely that the increased levels of RP mRNA 
are simply due to the presence of a higher percentage of dividing cells given the high regenerative rate of normal colon epithelium. Therefore, the higher mRNA levels could be the result of increased transcription or, alternatively, the result of decreased mRNA degradation. If the overexpression of some RPs could be an early event in the neoplastic progression of malignancy, their functional regulation would be closely associated with tumorigenesis. The demonstration of this hypothesis in a large series of different types of human tumors would help to detect malignancy at early stages. From a translational point of view, some RPs have been associated with resistance to chemotherapy. For example, the overexpression of L4, L5 and L36 in gastric cancer cells has been reported to be associated with multidrug resistance (including resistance to cisplatin) and to protect cells against vinblastine-induced DNA fragmentation $[6,7,138]$. In addition, L15 silencing reduces the growth rate of gastric cancer cells and sensitizes them to type I INF-induced inhibition of proliferation. This effect is explained by the physical interaction of L15 with p56, a gene encoding an antiviral protein [139], and is a potential extra-ribosomal function for L15.

Additional clues to establish the relevance of RPs in cancer formation and progression will come from the discovery, if any, of growth factors or mitogenic stimuli that would directly target a RP and cause a tumorigenic phenotype. The aforementioned effects of Myc targeting L24, which increases cap-dependent translation and prevents the switch to mitotic IRES-dependent translation, may be one example [75]. Endogenous mammalian rRNA genes can respond quickly to growth factor stimulation; therefore, the involvement of any RPs as early response genes in this context would not be surprising.

In humans, the overexpression of a single RP has not been conclusively proven to affect overall protein synthesis, as has been demonstrated in yeast. However, the levels of 
RACK1, a ribosomal protein, definitively affect overall protein synthesis. Overall, it is conceivable that some RPs act as major regulators of the important checkpoints related to ribosome biogenesis and/or proliferation, while other RPs may have a merely structural function in the ribosome. According to this model, a few RPs could be critical "sensors," as has been shown under certain circumstances (for example, under ribosomal or nucleolar stress). The RPs that may belong to this group are RPL5, RPL11 and RPL23 [140-142].

\section{RPs as growth inhibitory proteins}

Independent, non-coordinated changes in the expression pattern of an individual RP can occur under various cellular conditions, and in some circumstances, the overexpression of a single RP can provoke a growth inhibitory response. The fact that the overexpression of some RPs is associated with growth inhibition is apparently contradictory to the classical (and incorrect) assumption that a general increase in ribosome biogenesis and RPs occurs concomitantly with proliferation. An increasing number of proteins such as TGF- $\beta$, Notch, Runx, E2F, p21 ${ }^{\text {WAF1/CIP1 }}$ and KLF4 have a dual effect, functioning both as a tumor suppressor and as an oncogene; the same roles may be applicable to certain RPs [143]. Growing evidence shows that there is downregulation of RPs in specific human tumors and established tumorigenic cell lines (Table 1). First, in a loss-of-function genetic screen, we previously identified a cluster of RPs that regulate p53 function and act as possible tumor suppressor genes [144]. Moreover, reinforcing the role of RPs in senescence, in a senescence-induction model a number of genes encoding RPs were found the most strikingly downregulated, supporting a role of RP in the reduction of protein translation [145]. Second, the putative tumor suppressor function of some RPs has been demonstrated. For example, 
the overexpression of L41 arrests the cell cycle at the G1 phase in human lung carcinoma H1299 cells (which are p53-null), but in the presence of wild-type p53, L41 induces apoptosis. Experiments performed in vitro with different human carcinoma cells have shown that L41 acts as a suppressor of cell growth [146]. The enhanced expression of S29 induces apoptosis in the human non-small lung cancer cell line H520 by increasing p53 and bax and decreasing bcl-2, bcl-X $\mathrm{X}_{\mathrm{L}}$, survivin, and NF-K $\beta$ [147]. In combination experiments with anticancer drugs such as cisplatin or etoposide, the enhanced expression of S29 augments the chemotherapeutic response of these agents by mediating the down-regulation of bcl-2, bcl- $\mathrm{X}_{\mathrm{L}}$ and survivin [148]. Third, array studies show that RPL27, RPL37A and RPL41 are downregulated in cell lines that are derived from nasopharyngeal carcinomas [149]. Fourth, a loss of heterozygosity (LOH) on chromosome $3 p$ (where the RPL14 gene is located) occurs frequently in esophageal cancer. $\mathrm{LOH}$ of this locus has been observed in $43 \%$ of tumors, and decreased expression of the RPL14 gene has been detected in $63 \%$ of squamous cell carcinomas [15]. When examining dysplasia samples from the same patients, $47 \%$ displayed the same allelic loss as their corresponding tumors, and $41 \%$ exhibited microsatellite instability. If this observation holds true for a large series of squamous cell carcinomas, RPL14 could represent a potential novel molecular marker for the early detection of this largely unexplored cancer type at the molecular level. A complete picture of the relevant RPs in cancer will emerge from studies using knock-out mice for different RPs and the performance of next-generation sequencing (deep sequencing) in human tumors.

\section{RP deficiency}

The biological and physiological effects of any RP deficiency vary among species. In mammalian cells, an imbalance of RP levels or the absence or malfunction of any RPs 
has been shown to lead to ribosomal stress. The depletion of a single RP induces a decrease in the level of the other RP of the same ribosomal subunit with deleterious consequences for cell survival [150]. Consequently, the imbalanced production of both subunits provokes a block in rRNA maturation [150]. Moreover, mutated RPs assemble very poorly into ribosomal subunits [151]. Importantly, the best characterized response to ribosomal stress is mediated by the tumor suppressor $\mathrm{p} 53$. The overexpression of L5, L11 or L23 triggers p53 accumulation and activation, inducing a p53-dependent $\mathrm{G}_{1}$ arrest. Importantly, ribosomal stress is intimately coupled to cell cycle progression and proliferation via the regulation of the RPs-MDM2-p53 pathway. In principle, the RPMDM2 interaction blocks the E3 ubiquitin ligase function of MDM2, which results in p53 activation. This is the case for the following RPs: L5, L11, L23, L26, S3, S7, S14, S25, S26, S27, RPS27A and S27L, which bind to the oncoprotein HDM2 and block the HDM2-p53 interaction, increasing the half-life of p53 protein [72, 140-142, 152-160]. This observation supports the idea that free RPs (those that are not coupled to ribosomes) are not immediate targets for degradation (Figure 4). The depletion of L22, S14 or S19 causes p53 activation with a response that varies between cell cycle arrest, senescence or apoptosis depending on cell types and context [161-164]. For example, the ribosomal stress pathway is crucial in wild-type cells, cancer cells, and embryonic stem cells, and may also be a relevant cellular checkpoint during embryogenesis [165]. Moreover, S15, S20 and L37 regulate the MDM2-p53-MDMX network [166]. Therefore, the RP-MDM2 interaction is a genuine p53 stress-signaling pathway that is activated by aberrant ribosome biogenesis and is essential for safeguarding against oncogenic insults, such as Myc activation [167].

The inhibition of rRNA transcription by actinomycin D or 5-fluorouracil stabilizes p53 via the RPs and induces the inactivation of MDM2 [168]. Interestingly, the sites in 
HDM2 for L23 binding are different from those required for L11 binding, which explains the formation of a multi-protein complex. This fact suggests that a synergistic role of several RPs might result in p53 activation. Moreover, the mutual protection of L5 and L11 from degradation is essential for p53 activation upon ribosomal stress [169]. However, RP-MDM2 interactions are not the only interactions that activate p53. For example, L26 interacts with the 5'-UTR of p53 mRNA and increases the p53 translation rate. The overexpression of L26 modulates p53 protein levels and enhances p53 induction after DNA damage; this effect is blocked by the downregulation of L26 by siRNA [170]. Considering that the p53 5'-UTR predicts a highly structured stem loop that would be expected to inhibit translation, RPL26 may bind to this region and exert its positive effects. Moreover, S26 induces p53 stabilization and activation via an L11-dependent mechanism, resulting in p53-dependent cell growth inhibition [171]. Upon S26 knockdown, the ability of p53 to transcriptionally activate its target genes in response to DNA damage is impaired. Importantly, the knockdown of S26 does not have a noticeable effect on nucleolar integrity, an observation that is consistent with its extraribosomal function [171]. In addition, S26 can interact with p53 independently of MDM2 and coexists in a complex with p53 and p300 [172]. In turn, L26 is a substrate of MDM2. MDM2 binds to L26 to polyubiquitinate and degrade it, leading to the inhibition of p53 translation. Overall, p53 is regulated by a subset of certain RPs, and in turn, certain RPs are essential for p53 function. It would be interesting to study which p53 mutations in human tumors compromise the RP-p53 interaction as described for other RPs $[89,120]$.

On the other hand, in vivo experiments have revealed key aspects of RPs. For example, in yeast, the absence of S25 affects the translation of specific IRES mRNAs [173]. Similarly, L38+/- mice exhibit homeotic transformations of the skeleton due to the 
translational alteration of a subset of Hox mRNAs [106]. In certain organisms, such as zebrafish, RP genes may be haploinsufficient tumor suppressors, meaning that reducing their activities by a factor of approximately two increases the likelihood of cancer [174]. The developmental arrest of T cells in L22-deficient mice is dependent upon multiple p53 effectors [175]. Additional evidence shows that the deletion of a single allele of certain RPs is incompatible with survival (Table 3). For example, in vivo, the deletion of one allele of RPS6 in mouse leads to embryonic lethality at day E12.5 [176]. The genetic inactivation of p53 bypassed this checkpoint and prolonged development until E12.5, when death was caused by the decreased expression of D-type cyclins, diminished fetal liver erythropoiesis and placental defects. Thus, a p53-dependent checkpoint is activated in vivo during gastrulation in response to ribosome insufficiency to prevent the improper execution of the developmental program [170]. The zebrafish model suggests that the entire p53 family, including p63 and p73, may be involved in the ribosomal stress response [162].

The depletion of any RP can affect pathways alternative to p53. For example, RP depletion in HeLa cells causes the downregulation of mTOR. In particular, the effects caused by S19 or S14 depletion are mediated by mTORC2 [177]. The Proto-oncogene serine/threonine-protein kinase pim-1 (PIM1) has also been shown to be involved in the response to ribosomal stress independently of p53 [178]. The RP L11 recruits miR24/RISC to repress Myc expression in response to ribosomal stress [70]. In addition, unpublished observations from our laboratory show that the $\mathrm{p} 16^{\mathrm{INK} 4 \mathrm{~A}} / \mathrm{pRb}$ pathway can also be activated in primary cells upon the activation of a senescence response due to the deficiency of a RP (Perucho et al., unpublished observations). Finally, the unfolded protein response (UPR), a known pathway activated upon misfolded protein accumulation, is sensed and transduced by three major endoplasmic reticulum- 
transmembrane effector protein kinases: Serine/threonine-protein kinase/endoribonuclease IRE1 (IRE1), Eukaryotic translation initiation factor 2-alpha kinase 3 (PERK) and activating transcription factor 6 (ATF6). Interestingly, the perturbation of the ribosome biogenesis pathway plays an essential role in coupling the UPR to cell cycle regulation; HDM2/p53 signaling is therefore a crucial crosstalk mediator between ribosome biogenesis and the cell cycle [179].

The involvement of RP complexes with signaling pathways other than p53 (Ras, PI3K or $\mathrm{pRb}$ ) would be an exciting theme of investigation. We propose a model in which several factors can provoke a ribosomal stress response, which may in turn signal through several downstream pathways according to the degree of the stress (for example, the knockdown of an RP or RP haploinsufficiency) (Figure 6).

Overall, the key question would be, which are the essential RPs that provide or contribute to a protective response against ribosomal/nucleolar stress? In spite of the burgeoning literature showing p53 as the primary responder to cellular stresses (irradiation, hypoxia, DNA damage, depletion of nucleotides, heat-shock and oncogenic signals) [180], increasing evidence suggests that p53 is one player in the stress response mediated by the joined cooperation of several proteins that are crucial to guarantee a full and effective biological response. As reviewed here, the role of RPs in this context is essential because the RP-MDM2-p53 pathway is functional in vivo and plays an important role as ribosomal stress checkpoint. The deregulation of some RPs in tumors could reflect a result of response to stress (e.g., ribosomal stress, the UPR, and others).

\section{RP transcripts}

Transcripts encoding RPs can be among the most abundant in the cell [181-183]. This observation holds throughout the evolutionary tree and may be expected given the 
ribosome abundance in many cell types. Nevertheless, a closer look into the ribosomal transcriptome reveals a high degree of complexity and points to the existence of diverse post-transcriptional regulatory circuits in ribosome biogenesis. While the modulation of translation has been known for some time to have a significant impact on RP expression in mammalian cells, as, likely, does mRNA stability [184-186], in the context of this section, we will discuss recent questions regarding this circuitry that are centered on pre-mRNA splicing.

Most eukaryotic transcripts are subject to the process of alternative splicing [187]. Through pre-mRNA splicing, the coding segments (exons) of a given RNA are identified and joined together to produce an mRNA, discarding the internal non-coding sequences (introns). Alternative splicing occurs when exonic identification is changed (usually in response to cellular cues), producing a different mRNA. Consequently, alternative splicing expands both the coding and the genetic potentials of eukaryotic genomes [188]. The genes that encode RPs produce several mRNA isoforms with different sequences [189]; therefore, how splicing affects ribosome biogenesis and its relevance to health are important questions that need to be answered.

The current evidence points to a regulatory role of alternative splicing in ribosomal function (as opposed to generating more diversity in RPs). Across evolution, the control of pre-mRNA splicing is used to maintain proper levels of particular RP mRNAs [190194], supporting the notion that pre-mRNA processing impacts ribosome biogenesis. However, to our knowledge, there is no clear evidence yet of functional RP isoforms that are generated by alternative splicing. There are several observations that can be made in this regard. First, alternative splicing is likely to impinge on the relative amount of productive RP transcripts [189] because there are mechanisms to degrade mRNA isoforms that do not encode a full-length protein [194, 195] (the levels of these 
mRNA isoforms have been found to be low in mammalian cells $[196,197])$. Consequently, the cell could use alternative splicing to modulate ribosome production beyond the feed-back inhibitory loops described above. Second, experiments in yeast showed that the use of identical (in amino acid sequence) RPs encoded by paralogous genes can lead to non-identical ribosomes by mechanisms that remain obscure [198, 199]. According to this model, alternative splicing could potentially produce transcripts that encode the same amino acid sequence but have different ribosomal properties. This intriguing picture remains mostly unexplored, especially in human cells. Third, some RPs have extraribosomal roles [99], and it can be hypothesized that these roles are altered by alternative splicing by changing either the concentration or properties of a given RP. Last, high-throughput analyses indicate that RPs are synthesized in excess in cervical carcinoma cell lines, with the surplus RPs being rapidly degraded [100]. This is an important result that needs to be fully understood. It is difficult to reconcile with both the auto-regulatory schemes and the direct consequences of changing the expression of some RP genes (as discussed elsewhere in this review), suggesting that it may constitute a sign of cellular stress. However, this observation nevertheless has to be taken into account when considering the possible effects of changes in the relative amounts of RP transcripts.

In addition, analyses of ribosomal components (which are essential to accurately follow RP gene expression) face particular problems. Given their abundance, the presence of RPs (assumed to be particularly "sticky" given their size, charge and low solubility) in non-ribosomal purified samples can be considered an artifact $[99,200]$. Similarly, the number of RP-encoding transcripts may be out of the accuracy range of the experiment (as is the case with rRNA). Notably, modern high-throughput transcriptome studies (such as RNA-Seq), while extremely powerful, are not well-suited to the ribosome 
[201]. In fact, these analyses are based on the unambiguous mapping of short sequencing fragments ("reads") to the genome; this approach cannot be used when there are different hits for each read because it would be biased towards the most predominant reads and underrepresented sequences might not be identified. A troublesome source of mapping ambiguity is caused by pseudogenes $[93,202]$. This is highly relevant to the study of the ribosomal transcriptome because on average there are 20 pseudogenes for each RP gene, and these pseudogenes cover most of their exons [203]. Therefore, tailored strategies are required, such as building custom reference genomes. RP-pseudogene expression can also be estimated [204].

Unpublished RNA-Seq data from our group show great variation in the amounts of mRNA corresponding to each RP, consistent with previous reports [183]. This observation contrasts with the fact that RPs are needed in equimolar amounts (one per ribosome) and supports the hypotheses of active post-transcriptional regulation of RP genes and/or high turnover of excess RPs. Alterations in either case are likely to have consequences for the cell because they lead to changes in RP levels. Furthermore, there is the additional possibility that some sets of ribosomes lack a particular RP; this change would alter the translation properties of the ribosome, as has been shown with ribosomes missing L38 [106]. This phenomenon is relevant to cancer and other diseases connected to cell growth because stressed cells could use altered ribosomes to better suit their translation needs. Thus, while RPs themselves may not trigger cancer, it seems likely that tweaking RPs could provide a selective advantage to cancer cells. Interestingly, the number of mutations found in cancer in genes encoding pre-mRNA splicing factors is increasing [205]. In many cases, the effect of these mutations on premRNA splicing is not known, but mutations in highly abundant transcripts, such as those encoding RPs, are likely to result in novel isoforms at levels that are high enough 
to have an impact on the cell. An accurate description of the splicing landscape of ribosomal protein transcripts in healthy and cancer cells is needed to be able to resolve these important questions. Consistent with this notion, a recent report highlights the relevance of the connection between the spliceosome (the splicing catalyst) and the ribosome. Fregoso et al. [200] have shown that the oncogenic splicing factor serine and arginine splicing factor 1 (SRSF1), which is overexpressed in many cancers, interacts with L5 in the nucleus to trigger p53 stabilization upon ribosomal stress. As previously mentioned, this event is essential to induce cell-cycle arrest and cellular senescence.

To conclude, while there is ample evidence that alternative splicing and ribosome biogenesis have the potential of working together for the benefit of a cell, how this synergy can occur during important processes such as tumor progression still remains to be explored.

\section{Clinical implications}

An important concept that has emerged in the last decade is that transcription can be therapeutically targeted. For example, rDNA transcription can be targeted with the small molecule CX-5461 to selectively kill B-lymphoma cells in vivo while maintaining a viable wild-type $\mathrm{B}$ cell population. The therapeutic effect is a consequence of nucleolar disruption and the activation of p53-dependent apoptotic signaling [206]. This effect is mediated by the inhibition of Pol I, an enzyme that has also been considered as a therapeutic target in cancer. Moreover, translation has been the focus of pharmacological intervention. For example, eIF4E, a transcription factor that causes transformation, is a susceptible pharmacological target [207, 208]. mTOR signaling is currently one of the most important targets for drug development in cancer [209]. It is well known that sirolimus/rapamycin and its derivatives (known as rapalogs) act as powerful tumor-suppressive drugs in clinical trials due to their ability to inhibit the 
formation of mTORC1 complexes. For example, the drug SNS-032 targets mTORC1/mTORC2, neutralizing the subsequent activation of phosphorylation events in their downstream targets. This effect has been shown in acute myeloid leukemia cells [210]. Another drug, BEZ235, a multi-target pan-PI3K/dual-mTOR inhibitor, potently kills primary Myc-driven B-cell lymphomas and human cell lines bearing Myc translocations [211].

On the other hand, the term "ribosomopathies" refers to inherited pathologies or syndromes caused by defective genes involved in rRNA synthesis and/or processing (e.g., SBDS, DKC1, RMRP, NPM, TCOF1) and RP genes. All ribosomopathies are associated with increased cancer risk at different fold increases, depending on the disease. For example, germline mutations of the gene altered in dyskeratosis congenita (DKC1, which encodes dyskerin) have a direct effect on ribosome assembly. One of the functions of dyskerin is to act as a nucleolar protein associated with the snoRNPs involved in rRNA modification. The most striking finding of the direct effect of DKC1 mutations in cancer comes from the mouse model of dyskeratosis congenita, in which over $50 \%$ of mutant mice with DKC1 mutations develop tumors during their life [212]. On the other hand, mutations in RPs S7, S10, S15, S17, S19, S24, S26, S27A, L5, L11, L35A and L36 are found in Diamond-Blackfan anemia (DBA) patients. In fact mutations in these RPs have been identified in approximately $50 \%$ of DBA patients, and DBA mutations have not been discovered in any genes other than RPs. For example, a total of 77 different mutations have been described within the RPS19 gene [213]. The central defect in DBA patients is an enhanced sensitivity of hematopoietic progenitors to apoptosis [214]. This type of anemia involves the dysregulation of cancer-related genes and is associated with an increased risk of patients to develop leukemia [215, 216]. Interestingly, mutations in several RPs that are crucial for the p53 response to 
ribosomal stress and that interact with HDM2 (S7, L5 and L11) have been found in DBA patients. An in vitro DBA experimental model shows that the depletion of S19 or L11 in erythroblasts alters the translation of specific IRES mRNAs, suggesting that defective ribosomes are associated with the translation of specific mRNAs [217]. Patients with another disease, the 5q-syndrome (an independent subtype of myelodysplastic syndrome), have severe macrocytic anemia, but in this disease, a relatively low percentage of the patients develop leukemia (10\%). In 5q-syndrome patients, one allele of the RPS14 gene is deleted, causing RPS14 haploinsufficiency as a general characteristic of this disease [218].

It will be important to identify novel regulators of ribosome biogenesis and their respective impact on tumor pathogenesis. Given the fact that major oncogenes and tumor suppressor genes (HDM2, p53, etc.) modulate stress responses by their association with RPs, the discovery of the sensor molecules of ribosomal/nucleolar stress, including the identification of key RPs, will be of particular interest. Remarkably, the loss of the ability of cells to mount an effective stress-response makes cells more tumorigenic. It would also be interesting to explore if the HDM2 mutations that occur in human tumors compromise the ability of HDM2 to form complexes with RPs and to what extend such interaction influences p53 activation as shown in mice and the DBA model. Modern drugs that act to restore the ribosomal stress pathway may represent a promising non-genotoxic chemotherapeutic treatment against malignant tumors. In this regard, some anti-cancer therapeutic approaches act through RPs. For example, some drugs influencing RPs through post-transductional modifications (Figure 7) may represent a new drug approach. In this regard, drugs blocking the interaction of L26 with MDM2 would be promising as p53 inducers in tumors with wild-type p53. Moreover, specific hormone-related therapies such as gonadotropin-releasing hormone 
$(\mathrm{GnRH})$ analogues, which are used for the treatment of breast, prostate, and ovarian cancer, exert their anti-proliferative function through the downregulation of P1 and P2 [219]. Interestingly, curvularol, a specific cell-cycle inhibitor of mammalian cells, was demonstrated to be effective against L3 in yeast but ineffective in L3 mutant yeast [220]. Lastly, the fact that the expression levels of some RPs have been detected in early tumors while others have been associated with the transformation process or with a metastatic phenotype [221] suggests the existence of a tumor-specific RP profile associated with each malignancy that remains to be deciphered. Importantly, the fact that mutations in the RP genes RPL5 and RPL10 have been recently detected in cancer (in patients that were unrelated to any heritable disease) reinforces the importance of RPs as direct mediators of tumorigenesis [222].

\section{Conclusions}

The activation of Pol I and Pol III is a crucial requirement for cell proliferation, and these enzymes increase in response to oncogenic ERK, mTOR or Myc. In contrast, the tumor suppressors Rb, p53 and ARF exert a negative control of Pol I transcription and rRNA production. Thereby, deregulating the opposing activities that control rRNA synthesis may accompany tumor formation.

An increase in proliferation does not necessarily reflect an overexpression of all RPs. Equally, a cell cycle arrest does not necessarily involve a downregulation of all RPs. Therefore, RP control is a tightly regulated process in the cell.

While the classical/canonical role of many RPs is mainly structural, some of them have a dual role participating in the control of specific pathways (i.e., RPs-MDM2-p53). We suggest that the extraribosomal functions of some RPs could depend on the cell context 
and that tissue specificity could be more of a determinant than the ribosomal function of a given RP in the stress-response and/or proliferative status of the cell.

The recent data showing the stress-induced release of nucleolar proteins to carry out regulatory functions reinforce the idea that nucleoli orchestrate a chain of events that the cell uses to properly respond to stress signals. The activation of a ribosomal/nucleolar stress checkpoint, where RPs have a crucial role as "sensors," may play an important role in fundamental biological process, such as development, senescence, proliferation or aging.

Overall, the observations that: a) the activation of oncogenes and/or tumor suppressor genes is associated with the expression of some RPs, b) RPs are involved in the ribosomal stress response and in the DNA damage response and c) mutations in certain RP genes have been associated with cancer support the hypothesis that the dysfunction of RPs can be a direct cause of cancer formation.

\section{Acknowledgments}

We are grateful to the members of the Pathology Department at the Hospital Vall d'hebron. We especially thank J.A. Leal for scientific support in the review of the manuscript. The current study, including the contract of M. E. Lleonart, was supported by grants from the Ministerio de Sanidad (FIS PI12/01104. 


\section{Figures}

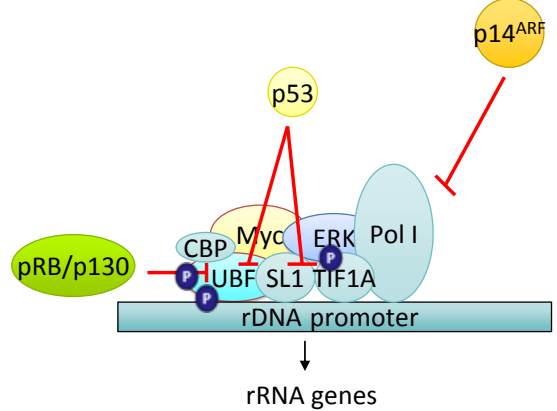

CKII

CDK4/Cyclin D1

CDK2/Cyclin E \& A

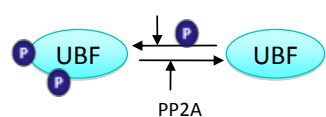

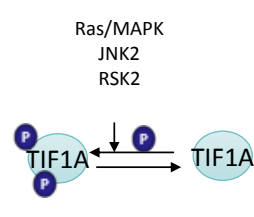

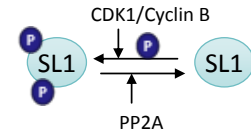

Figure 1: Oncogenes and Tumor suppressor genes control ribosomal biogenesis.

The classical tumor suppressor p53 controls Pol I transcriptional activity preventing the interaction between UBF and SL-1. Moreover, it inhibits Pol III transcription by binding to the transcription factor TFIIIB. The $\mathrm{Rb}$ family also plays a role in the inhibition of rRNA synthesis and Pol III transcription, when $\mathrm{pRb} 2 / 130$ and $\mathrm{pRb} 1 / 105$ bind to UBF and TFIIIB, respectively. Recently it was shown that $\mathrm{p} 14^{\mathrm{ARF}}$ interacts with the Pol I transcription termination factor TTF-I, preventing its localization to the nucleolus and inhibiting rRNA synthesis. Oncogenes, in contrast, have the opposite effect and increase ribosome biogenesis. The principal enhancers of ribosome biogenesis are Myc and ERK1/2. Myc associates with SL-1, increasing Pol I transcription, and is also found associated with tRNA and 5S rRNA activating Pol III transcription, whereas ERK phosphorylates and activates UBF, TIF1A and TFIIIB. In addition to these factors, numerous elements control the phosphorylation state of the Pol I complex. 


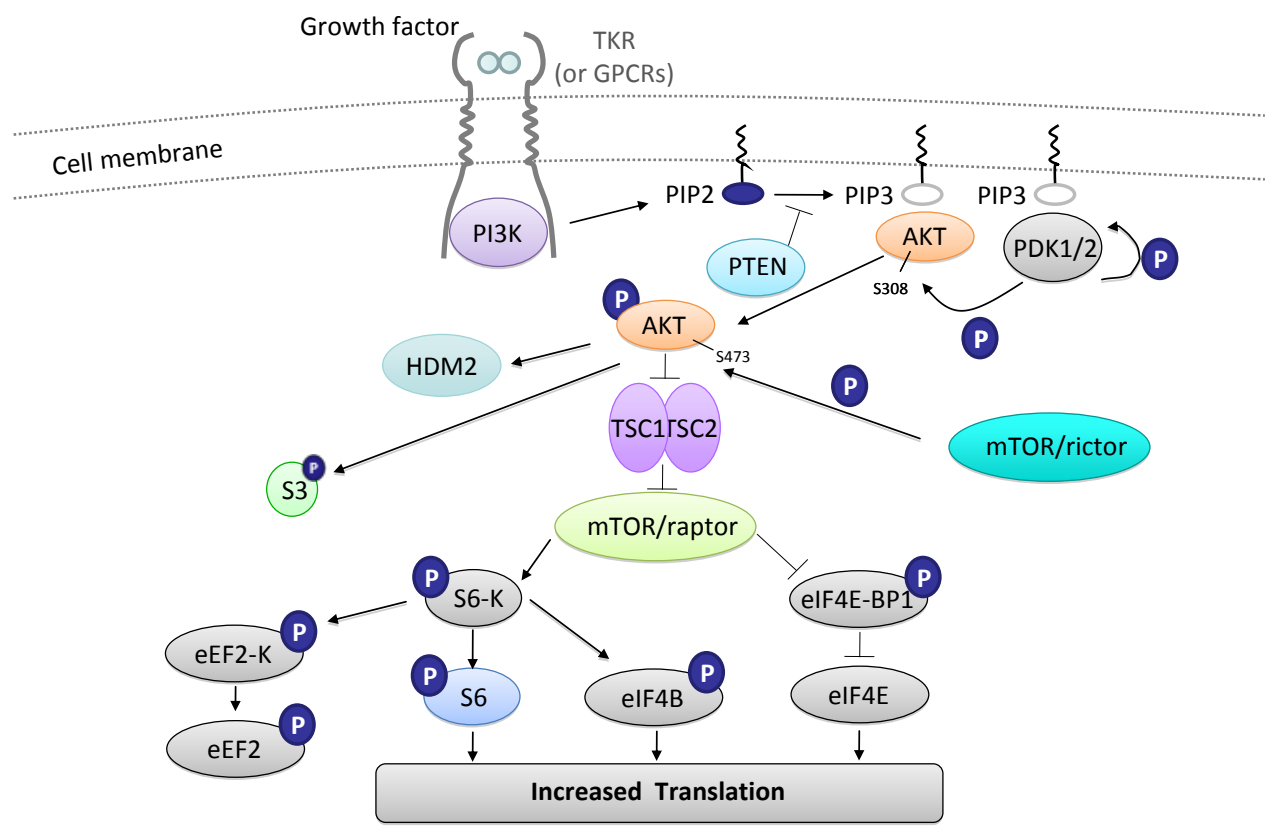

Figure 2: The PI3K/AKT/mTOR pathway has an important role in translation

Activated receptor tyrosine kinases (RTKs) recruit and activate PI3K, leading to increased phosphatidylinositol-3,4,5-trisphosphate (PIP3) levels. PIP3 recruits many proteins to the membrane, including $\mathrm{AKT}$ and phosphoinositide-dependent kinase (PDK1), by binding to their pleckstrin homology $(\mathrm{PH})$ domains. PDK1 is subsequently autophosphorylated and phosphorylates AKT. Once active, AKT phosphorylates a range of substrates, such as the tuberous sclerosis complex (TSC) proteins 1 and 2, well-known regulators of mTOR. The activation of TSC1 and TSC2 results in cellular growth, survival, and proliferation. Additional AKT targets related to the ribosomal stress response are HDM2, which controls the state of p53, and RP S3. 


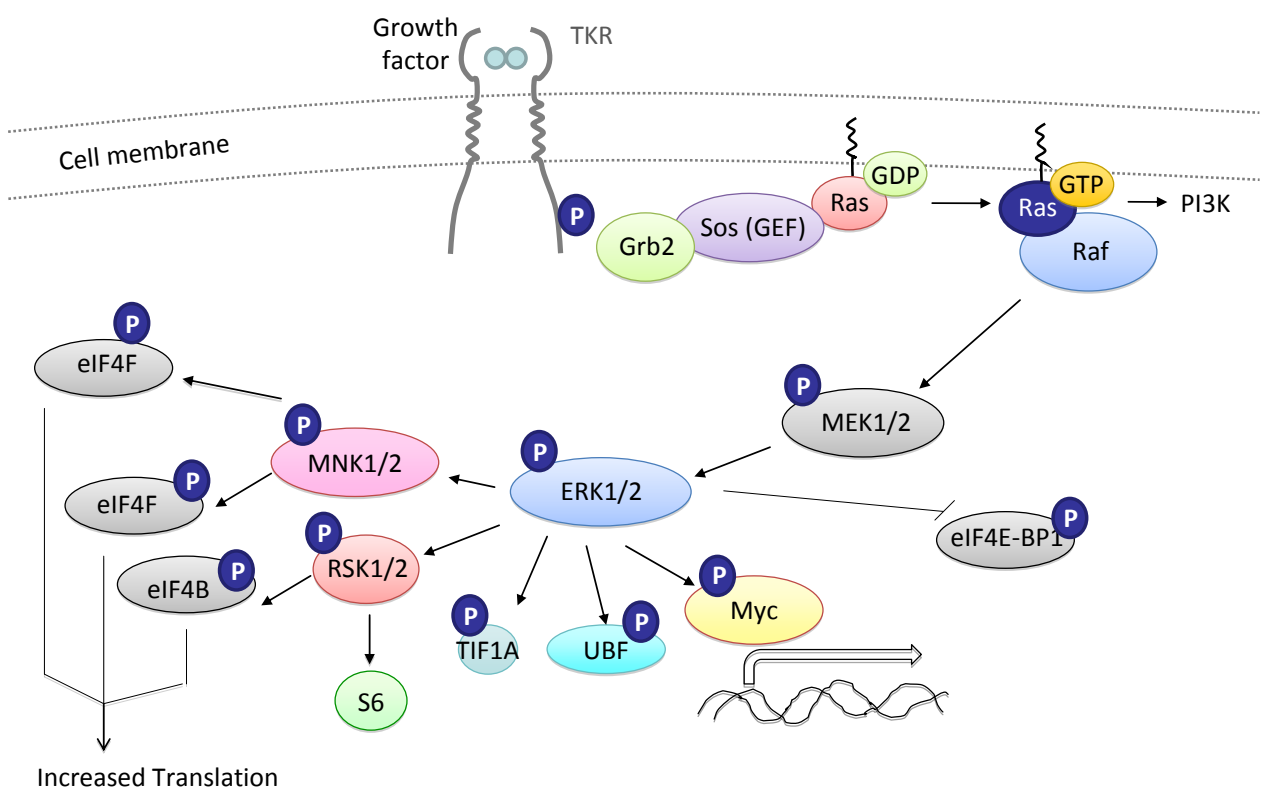

Figure 3: Effects of the Raf/Ras pathway on ribosome biogenesis.

The binding of epidermal growth factor (EGF) induces receptor dimerization and autophosphorylation on tyrosine residues. The phosphotyrosine residues of EGFR function as docking sites for signaling molecules, such as the Grb2-SOS complex. This complex activates the small G-protein Ras by stimulating the exchange of guanosine diphosphate (GDP) for guanosine triphosphate (GTP). This exchange elicits a conformational change in the Ras protein, enabling it to bind and recruit Raf to the cell membrane from the cytosol. Then, Raf-1 phosphorylates and activates MEK1/2, which in turn activates extracellular-signal-regulated kinase (ERK). ERK1/2 has many substrates in the cytosol that play an important role in translation and also can enter into the nucleus to control gene expression by directly phosphorylating multiple transcription factors. 


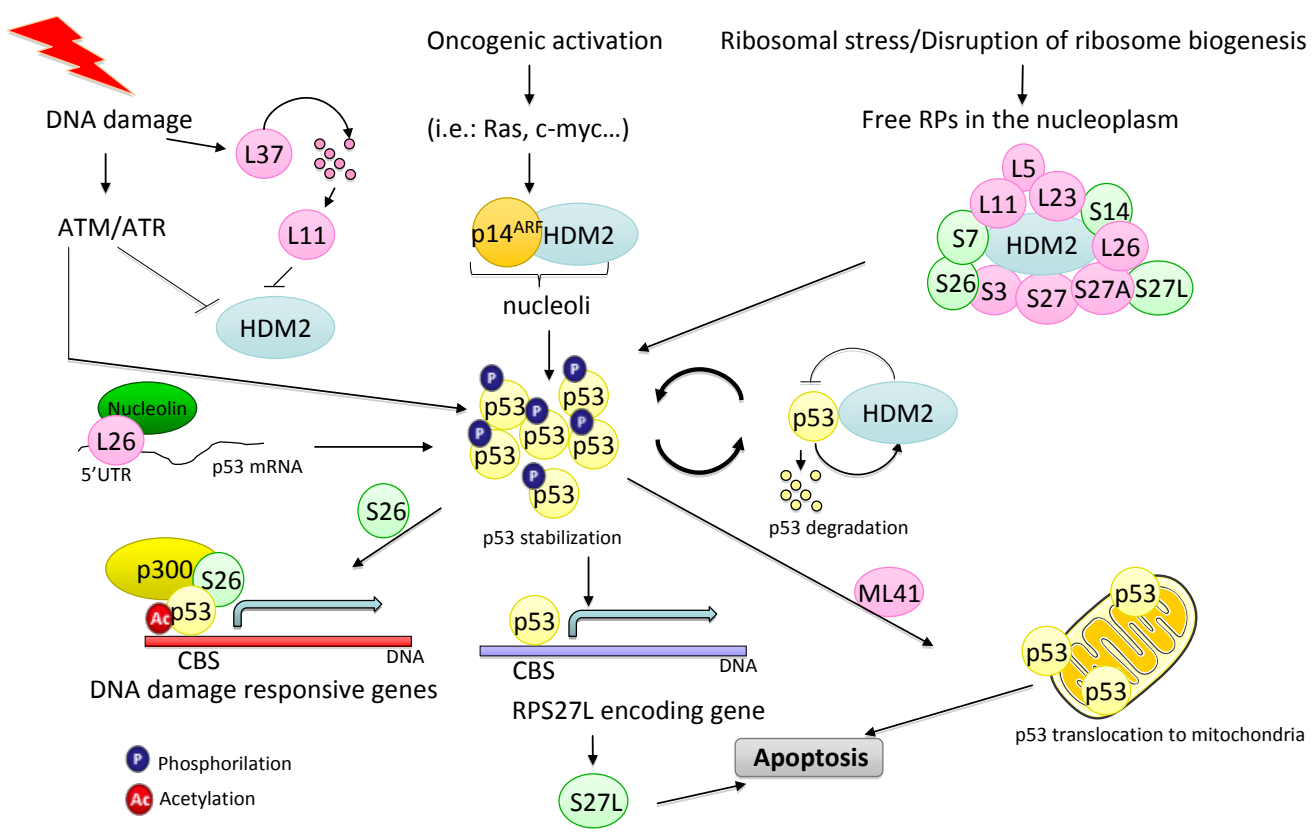

Figure 4: Different types of stress involve RPs to activate p53 tumor suppressor protein.

When oncogenic activation occurs, the main factor responsible for activating p53 is the tumor suppressor $\mathrm{p} 14^{\mathrm{ARF}}$, which binds to HDM2 and inhibits its E3 ubiquitin ligase activity against p53 and consequent degradation. Upon DNA damage, the canonical ATM/ATR kinase pathway is activated, affecting the ability of HMD2 to bind to p53 and regulate their translation. L26 and nucleolin play an important role in binding to the 5' untranslated region (UTR) of p53 mRNA to stimulate p53 activation. An alternative DNA damage pathway leads to the proteasomal degradation of L37, ensuring the L11dependent stabilization of p53 by binding and inhibiting HDM2. Upon ribosomal stress or deregulation of ribosome biogenesis, several free RPs can interact with HDM2 in the nucleoplasm, also resulting in the stabilization of p53. Once p53 is stimulated, it initiates the transcription of responsive genes. S26 participates in this process, which regulates p53 transactivation activity, enhancing its interaction with p300 and affecting p53 acetylation. P53 can also regulate the transcription of RPs such as S27L, which promotes apoptosis (the S27L protein only differs from S27 by three amino acids). RPs located in the mitochondria can also affect the function of p53. Mitochondrial RP 41 stabilizes p53 and enhances its translocation to the mitochondria, inducing p53's role in apoptosis. 

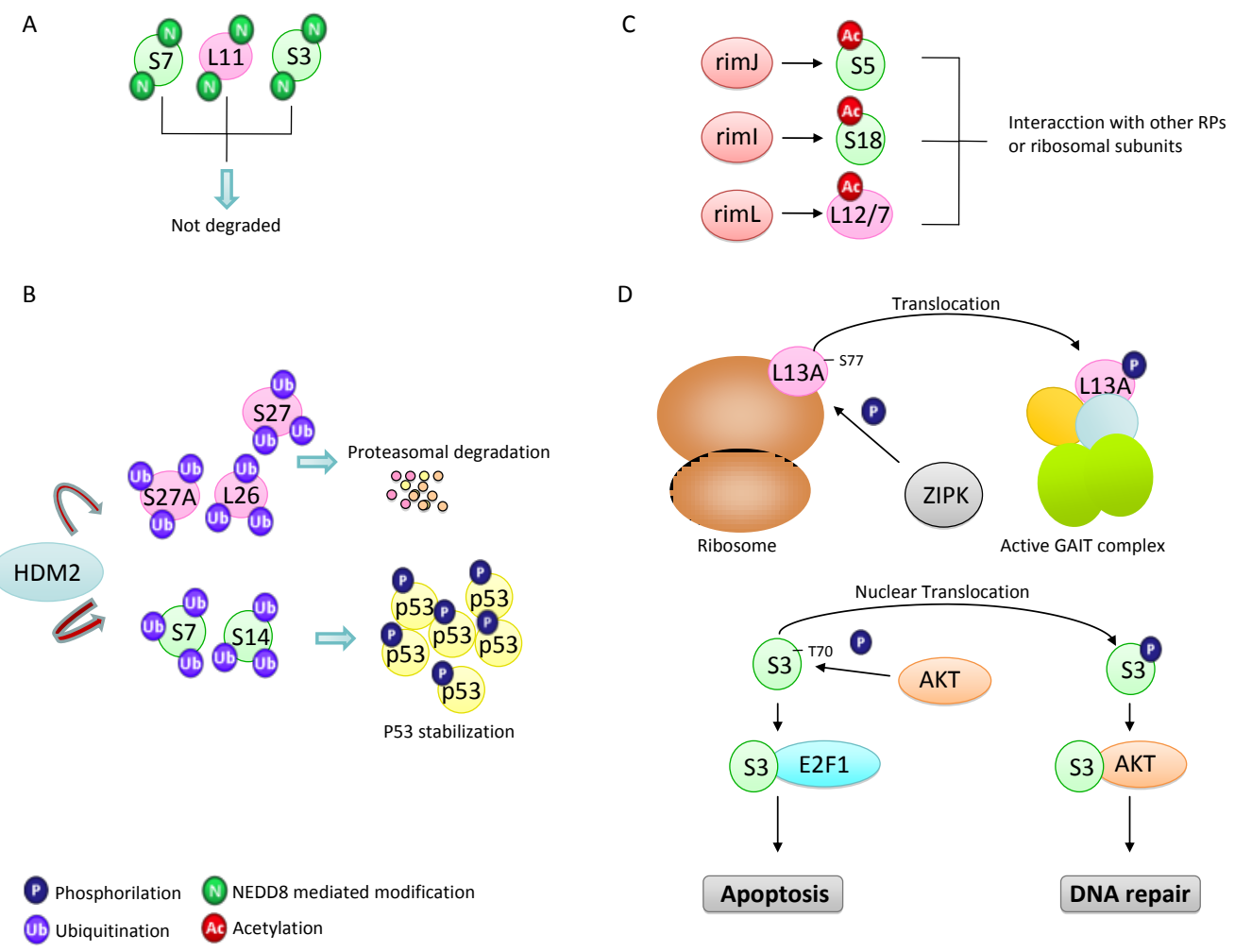

D
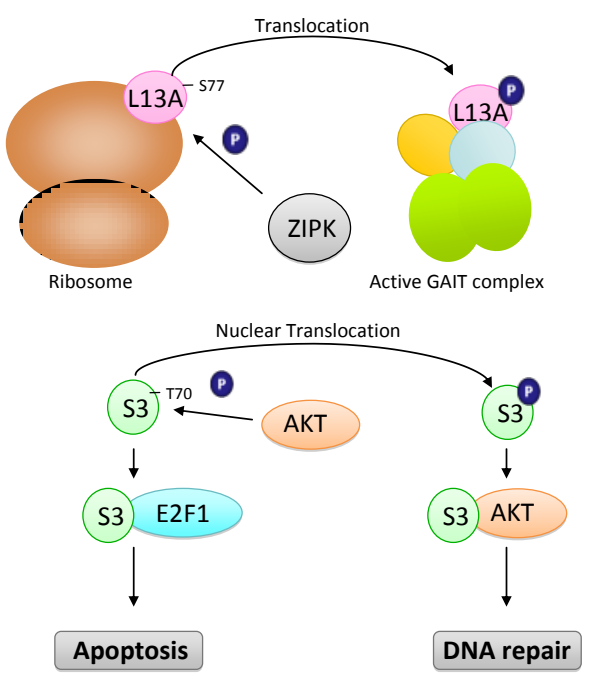

Figure 5: Posttranscriptional modifications of RPs allow them to acquire different functions.

A) The neddylation of RP S3, S7 and L11 by the ubiquitin-like molecule NEDD8 protects them from proteasome-mediated degradation.

B) A double role of ubiquitination from HDM2 has been observed for RPs; while the RPs S27A, S27 and L26 are ubiquitinated for proteasomal degradation, ubiquitinated S7 and S14 are not degraded and prolong p53 stabilization and activation.

C) In E. coli, it has been shown that N-acetylation of S5, S18 and L7 by Nacetyltransferases allows these RPs to interact with other RPs or ribosomal subunits.

D) RPs have different roles depending on their phosphorylation state. For example, when L13A is phosphorylated by zipper interacting protein kinase (ZIPK), it translocates from the ribosome to be assembled into the IFN-gamma-activated inhibitor of translation (GAIT) complex. This complex controls translation of a subset of inflammation-related proteins. Another example is S3; nonphosphorylated S3 is located in the cytoplasm, where interacts with E2F1. Upon S3 phosphorylation by AKT, S3 binds to AKT and accumulates in the nucleus, resulting in an increase of DNA repair activity. 


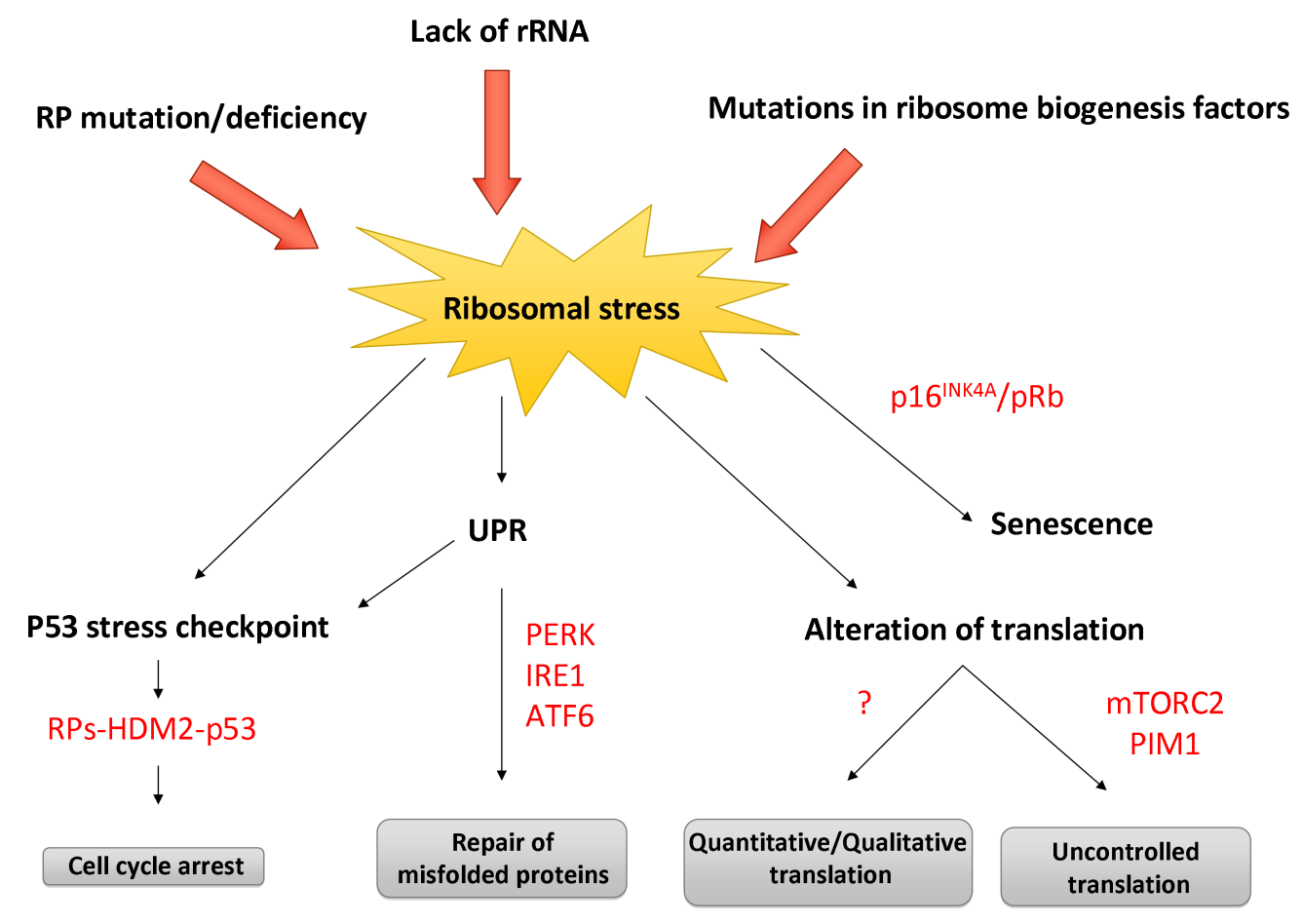

Figure 6: Ribosomal stress inducers

Different conditions, such as lack of rRNA, mutations in the elements involved in ribosomal biogenesis, or the absence of RPs lead to ribosomal stress. Under ribosomal stress conditions, several mechanisms are activated: Free RPs block the HDM2-p53 interaction, increasing p53 protein accumulation, which in turn results in the inhibition of cell proliferation. A lack of RPs can have an effect on proper protein folding; the accumulation of misfolded protein can initiate the unfolded protein response (UPR) pathway, transduced by three transmembrane effector proteins located in the endoplasmic reticulum: Serine/threonine-protein kinase/endoribonuclease IRE1 (IRE1), Eukaryotic translation initiation factor 2-alpha kinase 3 (PERK) and activating transcription factor 6 (ATF-6). This response can, on one hand, induce a p53-dependent cell cycle arrest or, on the other hand, initiate mechanisms involved in the repair of misfolded proteins. Alterations in translation can also involve additional responses, such as mTORC2 or PIM1 stimulation. Additional mechanisms associated with ribosomal stress can induce senescence, permanently blocking cell growth. 

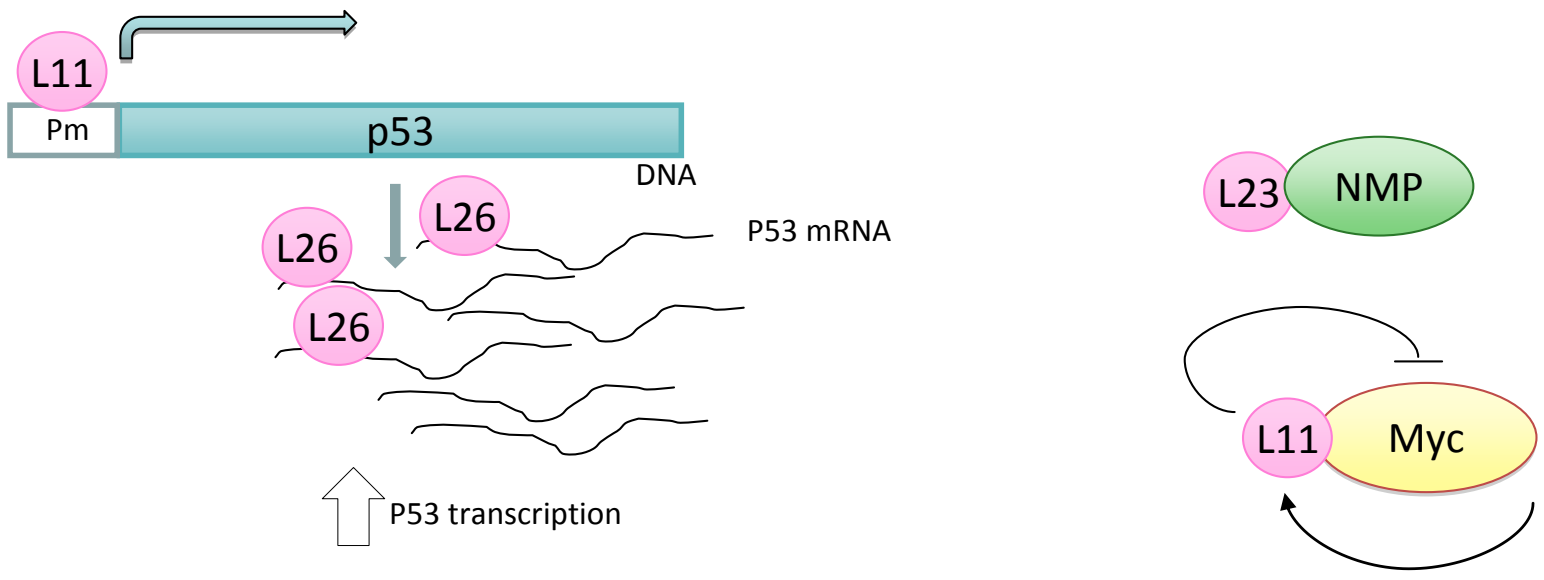

Figure 7: RPs can act at various levels. RPs can bind to gene promoters at the DNA level, facilitating the translation of specific genes, as is the case of L11 controlling p53 translation. Transcription can also be regulated by RPs; for example, the transcription of p53 can increase upon binding of L26 to p53 mRNA. Finally at the protein level, RPs can bind to other proteins to inhibit or activate them. 